\title{
AID downregulation is a novel function of the DNMT inhibitor 5-aza-deoxycytidine
}

\author{
Chiou-Tsun Tsai ${ }^{1}$, Pei-Ming Yang ${ }^{1}$, Ting-Rong Chern ${ }^{3}$, Shu-Hui Chuang ${ }^{1}$, Jung-Hsin \\ Lin $^{2,3,4}$, Lars Klemm ${ }^{5}$, Markus Müschen ${ }^{5}$ and Ching-Chow Chen ${ }^{1}$ \\ ${ }^{1}$ Department of Pharmacology, College of Medicine, National Taiwan University, Taipei, Taiwan \\ ${ }^{2}$ School of Pharmacy, College of Medicine, National Taiwan University, Taipei, Taiwan \\ ${ }^{3}$ Research Center for Applied Sciences, Academia Sinica, Taipei, Taiwan \\ ${ }^{4}$ Institute of Biomedical Science, Academia Sinica, Taipei, Taiwan \\ ${ }^{5}$ Department of Laboratory Medicine, University of California San Francisco, San Francisco, California \\ Correspondence to: Ching-Chow Chen, email: chingchowchen@ntu.edu.tw \\ Keywords: AID, 5-aza-CdR, Zebularine, DNMT1 \\ Received: August 23, $2013 \quad$ Accepted: November 23, $2013 \quad$ Published: November 25, 2013
}

This is an open-access article distributed under the terms of the Creative Commons Attribution License, which permits unrestricted use, distribution, and reproduction in any medium, provided the original author and source are credited.

\section{ABSTRACT:}

Activation-induced cytidine deaminase (AID) was originally identified as an inducer of somatic hypermutation (SHM) and class switch recombination (CSR) in immunoglobulin genes. However, AID can also cause mutations in host genes and contribute to cancer progression and drug resistance. In this study, molecular docking showed the interaction of free 5-aza-CdR and Zebularine (Zeb) with AID. However, only 5-aza-CdR-incorporated SSDNA bound to the active site of AID and inhibited AID expression through proteasomal degradation. 5-aza-CdR demonstrated cytotoxicity against AID-positive and -negative hematopoietic cancer cells. In contrast, Zeb exhibited a cytotoxic effect only in AID-negative cells due to its inability to inhibit AID expression. This differential effect might be due to the DNMT1 stabilization induced by AID, thus restricting the ability of Zeb to deplete DNMT1 and induce tumor suppressor genes (TSGs), such as p21, in AID-positive cells. Moreover, the in vivo anticancer effect of 5-aza-CdR but not Zeb in AID-positive hematopoietic cancer cells was demonstrated. The study not only displays the association of AID and DNMT1 and identifies a novel biological function of AID, but also provides novel information regarding the use of DNMT inhibitors to treat AID-positive hematopoietic cancers.

\section{INTRODUCTION}

AID, encoded by the AICDA gene, belongs to the apolipoprotein B-editing catalytic polypeptide (APOBEC) family and was originally described as a B cell-specific factor unique to activated germinal center B cells. During CSR, AID is recruited to the switch region to deaminate the nucleoside cytidine and convert it to uridine, causing DNA point mutations and double strand breakage [1]. This activity is essential for SHM and CSR, which generates immunoglobulin diversity after $\mathrm{V}(\mathrm{D}) \mathrm{J}$ recombination [2]. In contrast to the favorable role of AID in the immune system, AID can cause chromosomal translocations and/ or mutations in proto-oncogenes, thus promoting tumor formation [3]. For example, AID induces double strand breaks in the $c-m y c$ gene, resulting in its translocation to the $I g$ loci and uncontrolled expression of c-Myc in Burkett's lymphoma $[4,5]$.

AID also plays an essential role in the progression of Philadelphia-positive $(\mathrm{Ph}+)$ leukemias, including chronic myeloid leukemia (CML) and $\mathrm{Ph}+$ acute lymphoblastic leukemia (ALL) [6, 7]. The Ph chromosome originates from a translocation between the $c$ - $a b l$ on chromosome 9 and the $b c r$ gene on chromosome 22, leading to a $\mathrm{BCR} / \mathrm{ABL} 1$ fusion protein. The forced expression of the Abelson tyrosine kinase ABL1 can phosphorylate a wide range of substrates that regulate cell proliferation, differentiation, migration, survival, and DNA repair and drive the pathogenesis of $\mathrm{Ph}+$ leukemias [8]. Clinically, CML follows a triphasic pattern of chronic, accelerated, 
and blast crisis. The majority of patients $(85 \%)$ in the chronic phase will progress to the accelerated phase and blast crisis if untreated [9]. AID is expressed in a subset of CML patients in lymphoid blast crisis, which promotes the genetic instability of tumor suppressors and DNA repair genes through point mutations and copy number alterations. In addition, AID mutates BCR-ABL1, providing a rationale for the rapid development of imatinib resistance in blast crisis progression [6].

AID is also expressed in $\mathrm{Ph}+$ ALL patients, who show an increased mutation frequency of oncogenes and TSGs, such as MYC, BCL6, and p16, which may be relevant to the unfavorable prognosis in this subset of ALL [7]. Bone marrow isolated from wild-type and AID knockout mice were transduced with BCR-ABL1 to induce BCR-ABL1-driven ALL; then, ALL cells were transplanted into the mice. The mice engrafted with AID-/- ALL cells showed prolonged survival compared with those transplanted with AID+/+ ALL cells. Molecular analyses showed that AID-/- ALL cells had lower frequencies of amplification, deletion and point mutation in non-Ig genes, such as Pax5 and Rhoh, and failed to repress TSGs including Rhon, p21 and Blnk. The results indicate that AID may be an oncoprotein that accelerates the evolution of ALL through aberrant hypermutation and TSG downregulation [10]. A study has also shown that AID can induce B-lymphoma/leukemia in a bone marrow transplantation mouse model, and its activity to induce CSR and SHM is essential for lymphomagenesis [11].

The cytidine ribose nucleoside analogue 5-azaCR (5-azacytidine) was initially identified as a potential anticancer drug and was subsequently shown to be a DNA methyltransferase (DNMT) inhibitor [12]. After incorporation into DNA, 5-aza-CR is recognized by DNMT1 to form stable covalent protein-DNA adducts. DNMT1 are trapped and degraded, leading to rapid protein diminish as early as 24 hours treatment $[13,14]$. Eventually, DNA demethylation occurs, and then TSGs are induced to inhibit cancer cell proliferation [15]. 5-aza2-deoxycytidine (5-aza-CdR), the deoxyribose analogue of 5-aza-CR, was subsequently developed [16]. Cytidine deaminase (CDA) is a key enzyme in the pyrimidine salvage pathway, catalyzing the deamination of cytidine and deoxycytidine into uridine and deoxyuridine. CDA also deaminates cytidine analogues, including 5-aza$\mathrm{CR}$ and 5-aza-CdR, to reduce their stability $[17,18]$. Another DNMT inhibitor, 1-( $\beta$-D-ribofuranosyl)-2(1H)pyrimidinone (zebularine; Zeb), is a cytidine analogue that contains a 2-(1H)-pyrimidinone ring. Zeb was originally synthesized as a CDA inhibitor [19]. Because both CDA and AID can catalyze cytidine deamination, AID might also be targeted by cytidine analogues.

In this study, molecular docking analysis showed the interaction of both free 5-aza-CdR and free Zeb with AID. However, only 5-aza-CdR-incorporated single-strand DNA (ssDNA) bound to the active site of AID and inhibited AID expression through proteasomal degradation. The cytotoxicity of 5-aza-CdR was observed in both AID-positive and -negative hematopoietic cancer cells. In contrast, Zeb cytotoxicity was only observed in AID-negative cells due to its inability to inhibit AID expression. This differential effect might be due to the association and stabilization of DNMT1 by AID, thus restricting the ability of Zeb to deplete DNMT1 and induce TSGs, such as p21, in AID-positive cells. Moreover, the anticancer effect of 5-aza-CdR but not Zeb on AIDpositive hematopoietic cancers was also demonstrated in vivo. The study not only displays the association of AID and DNMT1 and identifies a novel biological function of AID, but also provides a novel role of a DNMT inhibitor for treating AID-positive hematopoietic cancers.

\section{RESULTS}

\section{Molecular docking of DNMT inhibitors to AID.}

A previous study showed that the DNMT inhibitor Zeb, a cytidine analogue, is a competitive inhibitor of CDA due to its lack of a 4-amino group on the cytosine (Fig. 1A) [20]. Because AID also catalyzes cytidine deamination, we hypothesized that cytidine analogues, such as 5-aza-CR, 5-aza-CdR, and Zeb, might inhibit AID. Molecular modeling simulation was performed to test this hypothesis. To our knowledge, the crystal structure of AID has not yet been solved. Because the sequences of AID and the APOBEC2 fragment shared 51.0\% similarity and 33\% identity (suppl. Fig. S1A), the AID structure was predicted based on the crystal structure of APOBEC2 [21] (suppl. Fig. S1B). The proposed binding modes of 5-azaCR, 5-aza-CdR, and Zeb are illustrated in Figure 1B. Free 5-aza-CR, 5-aza-CdR, and Zeb bound to the active site of AID and interacted with the zinc ion that is crucial for the enzymatic reaction by the 2',3' hydroxyl group and the 2' carbonyl group (Fig. 1B and suppl. S1C). Based on CDOCKER interaction energy, the binding capacity of 5-aza-CR (-37.7) and 5-aza-CdR (-35.95) was higher than that of Zeb (-27.312). These cytidine analogues are incorporated into nucleic acids after entering the cell membrane [13]. A previous study has shown that AID targets the immunoglobulin $\mathrm{H}(\mathrm{IgH})$ switch region, which contains 5'-AGCT-3' repeats in its core [22]. Therefore, 5'-AGCT-3' ssDNA, in which the cytidine base was substituted by the respective azacytidine (5'-AGazaC-T-3') or Zeb (5'-AG-ZebC-T-3'), was analyzed by molecular docking. The 10 predicted binding modes are illustrated in Figure 1C. Compared with 5'-AG-ZebC-T-3' (Fig. 1C, right panel), 5'-AG-azaC-T-3' was closer to the catalytic site of AID, and both of them inserted into the 
active site (Fig. 1C, left panel). The best insertion model showed that the substituted 5-aza-cytosine inserted exactly into the small active site (Fig. 1D), while the remainder of the ssDNA interacted with the polar surface (as indicated by the orange color) of AID (Fig. 1D, right panel). These findings suggest that only azacytidine-incorporated ssDNA could interact with AID.

\section{5-aza-CdR but not Zeb destabilizes AID through ubiquitin-proteasomal degradation.}

Once incorporated into DNA, azacytidine traps DNMT and triggers its degradation [14]. Because azacytidine- but not Zeb-substituted ssDNA interacted with AID (Fig. 1C), we proposed that 5-aza-CdR and 5-aza-CR would also trigger AID degradation. Therefore, the inhibitory effect of DNMT inhibitors on AID expression was examined in Burkitt's lymphoma Raji and Ph+ ALL SUP-B15 cells, which possess AID. 5-aza-CdR, 5-aza-CR, and Zeb all downregulated DNMT1 expression [13]; however, only 5-aza-CdR and 5-aza-CR inhibited
AID expression (Fig. 2A, 2B and suppl. Fig. S2A). However, AICDA mRNA was not significantly affected by 5-aza-CdR (Fig. 2C and 2D), indicating that 5-aza-CdR might inhibit AID expression through post-transcriptional regulation.

AID stability has been reported to be regulated through the proteasome degradation pathway [23]. To investigate how 5-aza-CdR downregulates AID, the cells were treated with 5-aza-CdR in the presence of the proteasome inhibitor MG132. Restoration of AID expression was observed (Fig. 3A, upper panel), suggesting the involvement of proteasomal degradation in this event. To further confirm this observation, AID protein stability was examined in the presence of cycloheximide. As shown in Figure 3A, lower panel, 5-aza-CdR reduced AID protein stability, which was reversed by MG132. Because proteasome degradation is usually triggered by polyubiquitination [23], nuclear AID ubiquitination was analyzed using an immunoprecipitation assay. The smear blotting was more intense after co-treatment with 5-aza-CdR and MG132 (Fig. 3B, left panel), indicating that 5 -aza-CdR enhanced AID polyubiquitination. AID

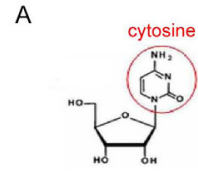

Cytidine

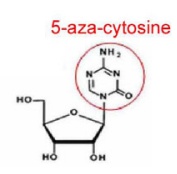

5-aza-CR

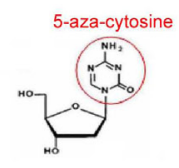

5-aza-CdR

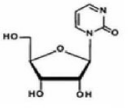

Zeb
B

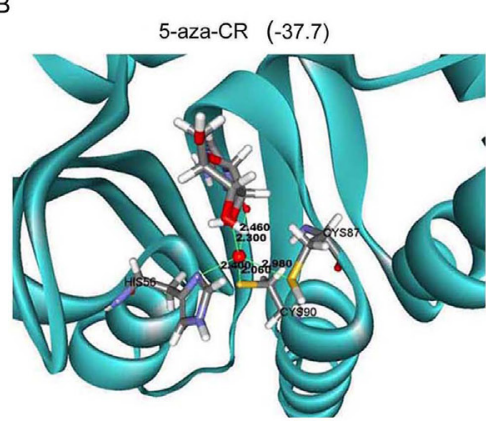

C Top ten binding poses of ssDNA
$C$ was replaced by 5-aza-CR or 5-aza-CdR (5'-A-G-azaC-T-3')

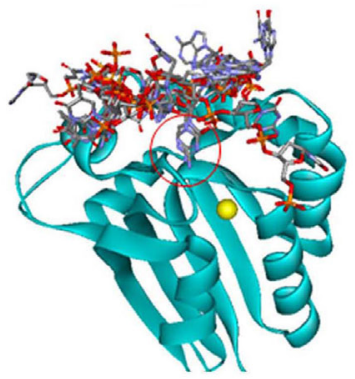

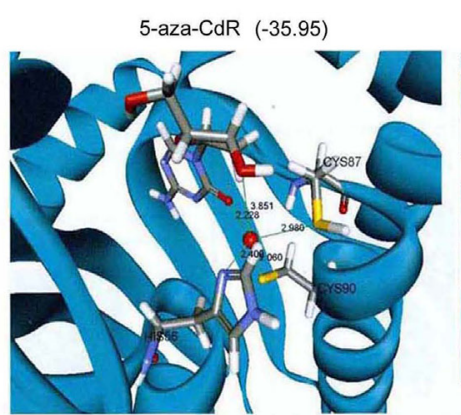

D

$C$ was replaced by Zeb (5'-A-G-ZebC-T-3')

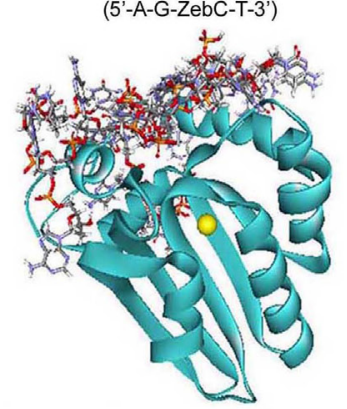

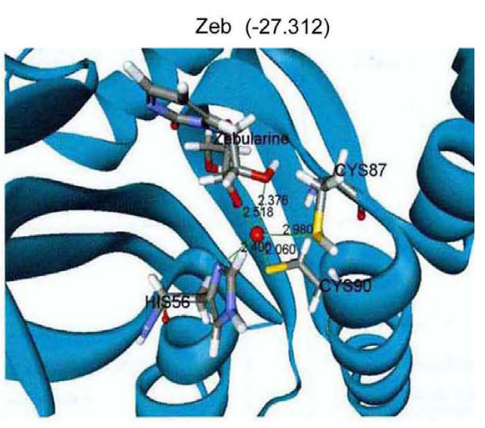

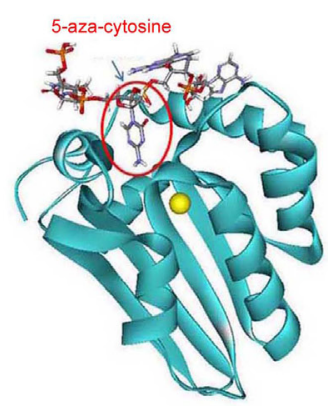

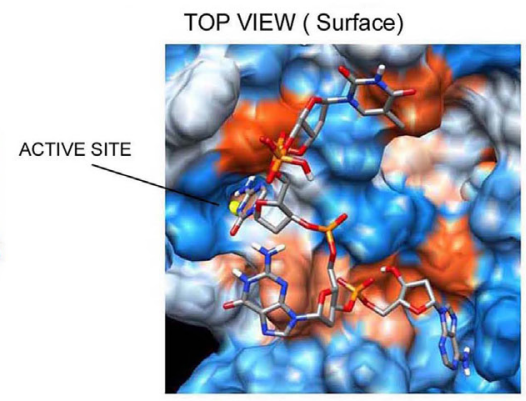

Figure 1: Molecular docking of DNMT inhibitors to AID. (A) Molecular structures of cytidine and its analogs. (B) The docking sites of DNMT inhibitors were analyzed using CDOCKER, and the best structures were selected based on the lowest CDOCKER INTERACTION_ENERGY for each ligand. (C) 5-aza-CdR and Zeb were incorporated into the DNA sequence (5'-AGCT-3') built with "Build and Edit Nucleic Acid" tools in Discovery Studio 2.55, and the binding modes were further analyzed. The top ten binding structures are shown. (D) The best insertion model is presented by a ribbon (left panel) and surface model (right panel). 
degradation has been reported to occur in the nucleus [23]; therefore, nuclear AID expression was examined. AID was substantially downregulated in the nucleus by 5 -aza-CdR (Fig. 3B, right panel). To confirm this finding, AID-negative CML K562 cells were transfected with flag-AID through electroporation, and stable clones were established. Nuclear flag-AID was downregulated by 5-aza-CdR but not Zeb, although total flag-AID was increased by 5-aza-CdR and Zeb (Fig. 3C, left panel). Immunofluorescence (IFA) also indicated the degradation of nuclear AID by 5-aza-CdR (Fig. 3C, right panel). Because AID is normally restricted in cytoplasm to prevent off-target deamination [24], a Crm1 inhibitor, leptomycin B (LMB), which accumulates AID in the nucleus [25], was further utilized to demonstrate the occurrence of this event in the nucleus (Fig. 3D).

\section{The role of AID expression in the cytotoxicity of DNMT inhibitors.}

Because 5-aza-CdR but not Zeb was found to induce AID degradation, the cytotoxicity of each agent was examined in hematopoietic cells expressing different AID levels. The cell viability of K562 (AID negative), Raji (high AID) and SUP-B15 (low AID) cells was assessed. Both 5-aza-CdR and Zeb inhibited the cell viability of K562 cells, while Raji and SupB15 cells were sensitive to 5-aza-CdR but resistant to Zeb (Fig. 4A and 4B). This differential effect indicated that AID might play an interfering role in the anticancer effect of DNMT inhibitors. To confirm this, the growth inhibitory effects of 5-aza-CdR and Zeb were examined in AID-knockdown Raji or AID-overexpressing K562 cells. The inhibition of cell viability by Zeb was enhanced in AID-knockdown Raji cells but attenuated in AID-overexpressing K562 cells (suppl. Fig. S3, Fig. 4C and 4D), while the inhibitory ability of 5-aza-CdR was not influenced by AID expression (Fig. 4C). These results suggest that 5-aza-CdR targets both AID-positive and -negative cancer cells due to its ability to deplete AID. To exclude the possibility that AID-mediated genetic mutations alter Zeb cytotoxicity, K562 cells were transiently transfected with AID. Consistently, the cytotoxicity of Zeb was attenuated by AID overexpression (Fig. 4D, right panel).

\section{AID interferes with the effect of DNMT1 inhibitor through stabilizing DNMT1}

DNMT inhibitors induce growth arrest by downregulating DNMT1 to promote the expression of tumor suppressor genes [15]. Because AID expression differentially influenced the cytotoxic effect of 5-aza$\mathrm{CdR}$ and Zeb, its role in DNMT1 inhibition was further examined. Both 5-aza-CdR and Zeb depleted DNMT1 in AID-deficient K562 cells, whereas the effect of Zeb was apparently attenuated in AID-positive Raji cells (Fig. 5A, left panel). Consistently, Zeb effectively downregulated DNMT1 in AID-knockdown Raji cells but not in AIDoverexpressing K562 cells (Fig. 5 A, right panel and 5B, left panel), indicating that the inhibitory effect of Zeb on DNMT1 might be attenuated by endogenous AID levels due to its inability to deplete AID. Similarly, the induction of the tumor suppressor gene p21 by Zeb but not 5-azaCdR was diminished in AID-overexpressing K562 cells (Fig. 5B, right panel).

A positive correlation between the protein but

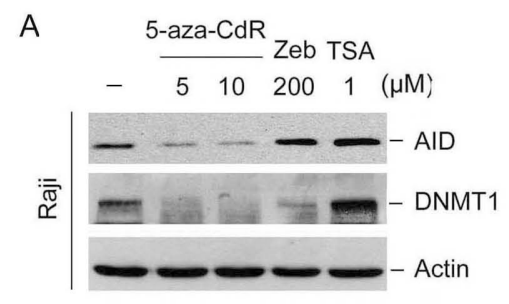

B

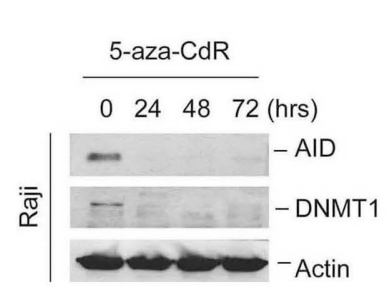

C

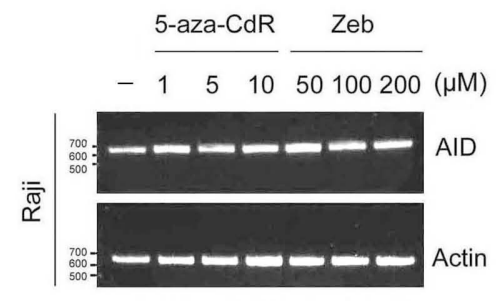

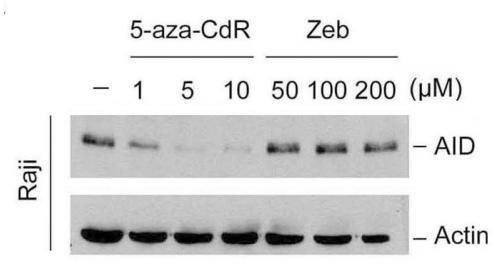

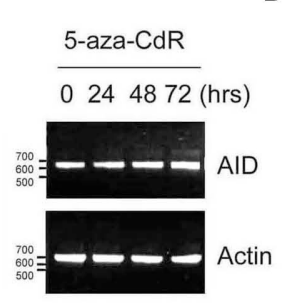

D

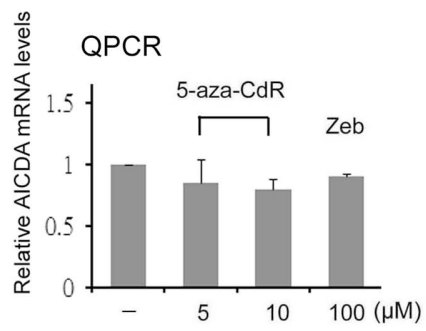

Figure 2: 5-aza-CdR downregulated AID. Raji cells and SUP-B15 were treated with 5-aza-CdR (1-10 $\mu \mathrm{M})$, Zeb (50-200 $\mu \mathrm{M})$, or TSA $(1 \mu \mathrm{M})$ for 4 days (A) or 5-aza-CdR $(5 \mu \mathrm{M})$ for 24,48 , and $72 \mathrm{hrs}(\mathrm{B})$. The protein expression levels of AID, DNMT1 and actin were analyzed through immunoblotting. (C) Raji cells were treated with 5-aza-CdR (1-10 $\mu \mathrm{M})$ or Zeb (50-200 $\mu \mathrm{M})$ for 4 days (left panel) or 5-aza-CdR $(5 \mu \mathrm{M})$ for 24, 48, and $72 \mathrm{hrs}$ (right panel). The mRNA levels of AICDA and actin were analyzed through RT-PCR. (D) Raji cells were treated with 5-aza-CdR $(5-10 \mu \mathrm{M})$ or Zeb $(100 \mu \mathrm{M})$ for 4 day. The relative mRNA levels of AICDA were analyzed through QRT-PCR 
not mRNA expression levels of AID and DNMT1 was observed in several stable clones of AID-knockdown Raji cells and AID-overexpressing K562 cells (suppl. Fig. S3A-S3C). AID overexpression also increased ectopic DNMT1 expression, indicating the positive regulation of these two enzymes at the protein level (suppl. Fig. S3D). Furthermore, DNMT1 stability was decreased in AID-knockdown Raji cells but increased in AIDoverexpressing K562 cells (Fig. 5C, left panel), and the inhibition by Zeb was attenuated by AID overexpression (Fig. 5C, right panel). These results indicate that AID may stabilize DNMT1 to prevent its degradation by DNMT inhibitors. Flag-AID ectopically expressed in K562 cells and endogenous AID in Raji cells were coimmunoprecipitated with DNMT1 (Fig. 5D, upper pamel), and the co-localization of AID and DNMT1 in Raji cells was demonstrated (Fig. 5D, lower panel). These results suggest that the association of AID and DNMT1 might stabilize DNMT1. The silencing of AID by shAID caused DNMT1 depletion and induction of TSGs, such as p21 and
Rassfla (Fig. 5E).

\section{5-aza-CdR inhibits cell growth in a mouse model.}

Because AID accelerates CML progression by causing imatinib resistance [6], the effect of DNMT inhibitors on KCL22+AID cells was investigated. Consistent with the results from K562 cells, Zeb- but not 5-aza-CdR-induced cytotoxicity and DNMT1 downregulation were partially attenuated by AID overexpression, and 5-aza-CdR also downregulated nuclear AID levels (Fig. 6A and 6B). KCL22 cells harboring AID fused to a luciferase reporter were intratibially injected into NOD/SCID mice to examine the in vivo anticancer effect of 5-aza-CdR and Zeb. After transplantation, the cells grew in the bone marrow and spread throughout the body; However, KCL22+AID cells displayed higher growth rate (83\%) than KCL22 cells $(42 \%)$ (suppl. Fig. S4). The tumor-bearing mice were then selected and treated with Zeb $(500 \mathrm{mg} / \mathrm{kg})$ or 5 -aza-CdR
A

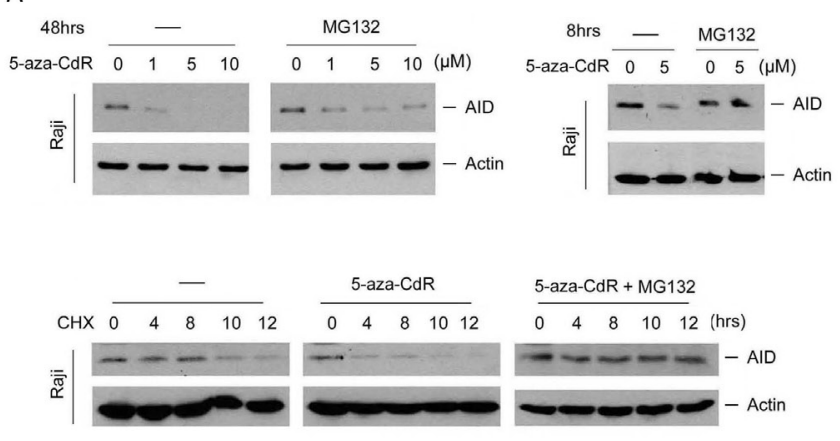

B

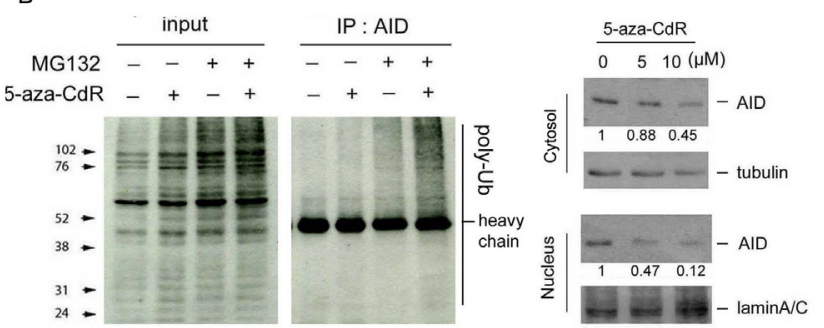

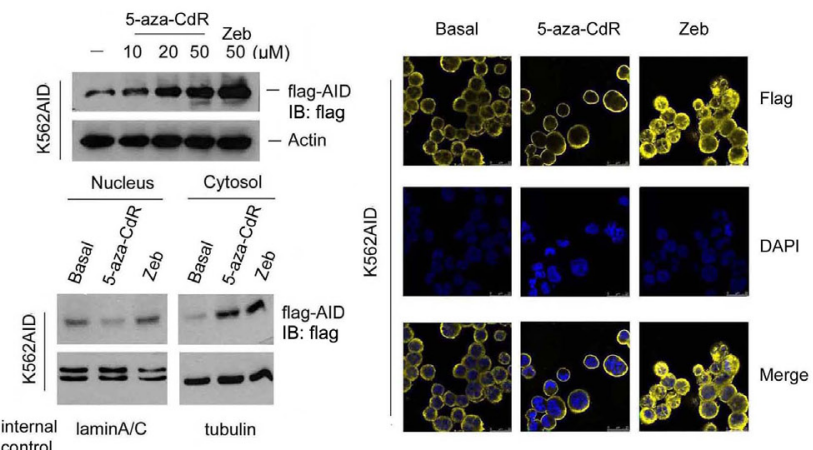
control

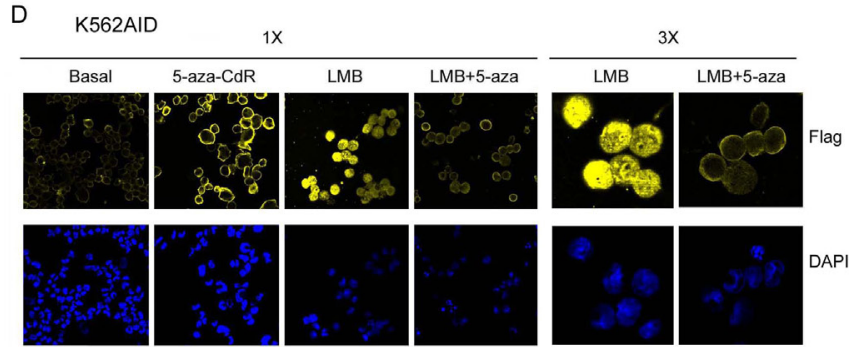

Figure 3; 5-aza-CdR reduced the protein stability of nuclear AID. (A) Upper-left panel: Raji cells were treated with 5-aza-CdR (1-10 $\mu \mathrm{M})$ for $40 \mathrm{hrs}$, and MG132 $(10 \mu \mathrm{M})$ was added for another $8 \mathrm{hrs}$. Upper-right panel: Raji cells were co-treated with 5-aza-CdR (5 $\mu \mathrm{M})$ and MG132 $(10 \mu \mathrm{M})$ for $8 \mathrm{hrs}$. The protein expression levels of AID and actin were analyzed through immunoblotting. Lower panel: Raji cells were pretreated with 5-aza-CdR $(5 \mu \mathrm{M})$ for $16 \mathrm{hrs}$. Then, the cells were exposed to cycloheximide $(20 \mu \mathrm{M})$ or cycloheximide/ MG132 $(10 \mu \mathrm{M})$ for 4, 8, 10, and $12 \mathrm{hrs}$. The protein expression levels of AID and actin were analyzed through immunoblotting. (B) Left panel: Raji cells were pretreated with 5-aza-CdR $(10 \mu \mathrm{M})$ for $19 \mathrm{hrs}$; then, the cells were exposed to MG132 (10 $\mu \mathrm{M})$ for 5 hrs. AID was immunoprecipitated, and AID ubiquitination was examined through immunoblotting. Right panel: Raji cells were treated with 5-aza-CdR $(5-10 \mu \mathrm{M})$ for $48 \mathrm{hrs}$, and the nuclear and cytosolic extracts were harvested. The protein expression levels of AID, tubulin, and lamin A/C were analyzed through immunoblotting.. (C) Upper-left panel: K562AID4 cells were treated with 5-aza-CdR (10-50 $\mu \mathrm{M})$ and Zeb $(50 \mu \mathrm{M})$ for $48 \mathrm{hrs}$. Lower-left panel: K562AID4 cells were treated with 5-aza-CdR $(10 \mu \mathrm{M})$ and Zeb $(10 \mu \mathrm{M})$ for $48 \mathrm{hrs}$, and the nuclear and cytosolic extracts were harvested. The protein expression levels of flag-AID and actin were analyzed through immunoblotting. Right panel: K562AID4 cells were treated with $10 \mu \mathrm{M}$ DNMT inhibitors for 4 days. The cells were fixed and stained with an anti-flag antibody and DAPI and subjected to confocal microscopy analysis. (D) K562AID4 cells were treated with 5-aza-CdR (10 $\mu \mathrm{M}), \mathrm{LMB}(5 \mathrm{ng} / \mathrm{ml})$, or both for $48 \mathrm{hrs}$. The cells were fixed and stained with an anti-flag antibody (yellow) and DAPI (blue) and subjected to confocal microscopy analysis. The images are shown at $1 \mathrm{X}$ and $3 \mathrm{X}$ magnification. 

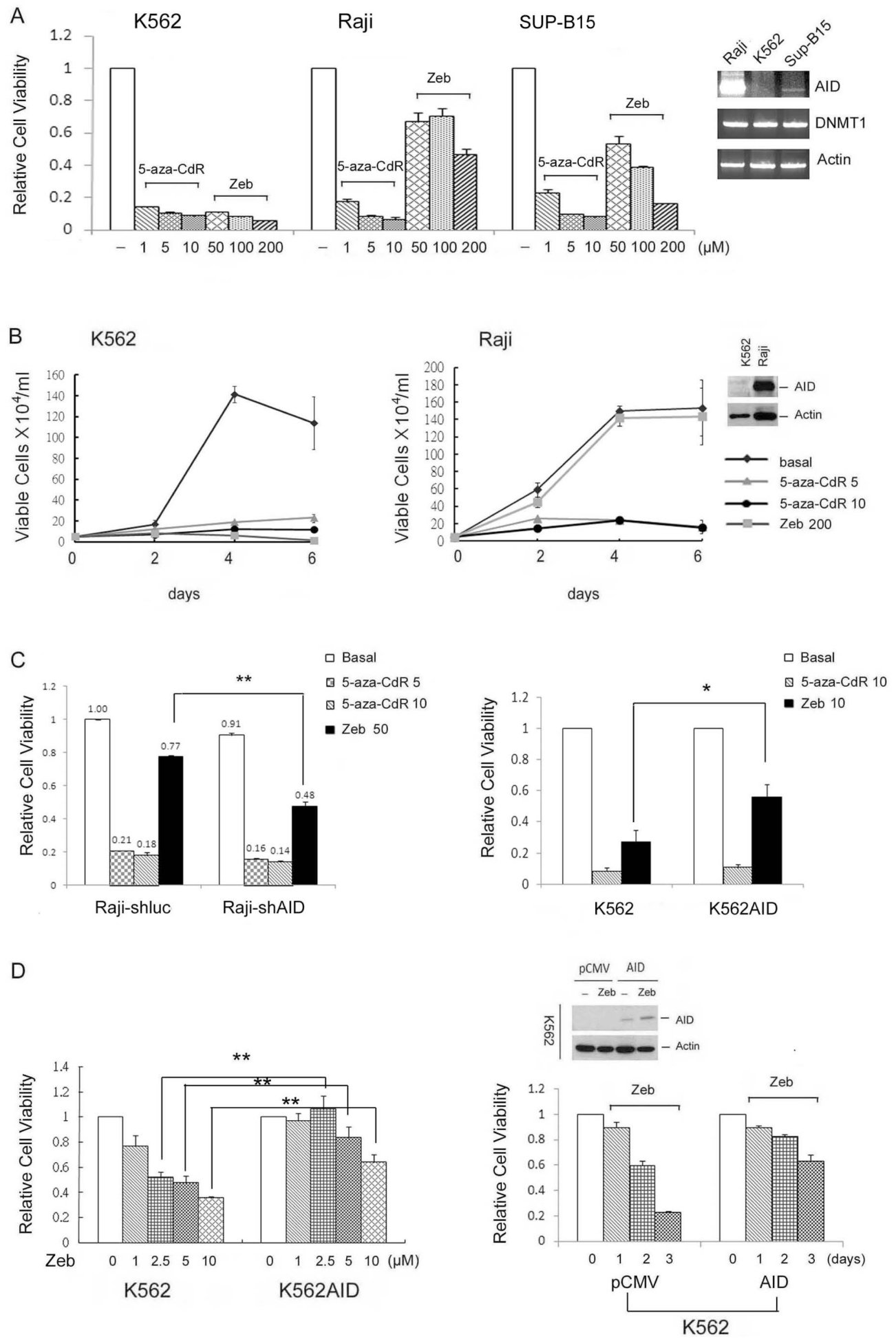

Figure 4: AID interferes with the cytotoxic effect of DNMT inhibitor in vitro. (A) K562, Raji, and SUP-B15 cells were treated with 5-aza-CdR $(1-10 \mu \mathrm{M})$ or Zeb $(50-200 \mu \mathrm{M})$ for 4 days, and cell viability was analyzed with the Alamar blue assay. Error bars indicate the mean \pm SD of three independent experiments. (B) K562 and Raji cells were treated with 5-aza-CdR (5-10 $\mu$ M) and Zeb (200 $\mu$ M) for 0-6 days, and cell viability was analyzed with the trypan blue assay. (C) Left panel: Raji-shluc and Raji-shAIDH1 cells were treated with 5 -aza-CdR $(5-10 \mu \mathrm{M})$ or Zeb $(50 \mu \mathrm{M})$ for 4 days, and cell viability was analyzed with the Alamar blue assay. **, p $<0.01$. Right panel: K562 and pooled K562AID cells were treated with 5-aza-CdR $(10 \mu \mathrm{M})$ or Zeb $(10 \mu \mathrm{M})$ for 4 days. Then, cell viability was analyzed with the Alamar blue assay. *, p $<0.05$. (D) Left panel: K562 and pooled K562AID cells were treated with Zeb (1-10 $\mu$ M) for 4 days. Then, cell viability was analyzed with the Alamar blue assay. ${ }^{*}, \mathrm{p}<0.01$. Right panel: K562 cells were transiently transfected with $\mathrm{pCMV}$ or $\mathrm{pCMV-}$ AID plasmids and exposed to Zeb $(5 \mu \mathrm{M})$ for 3 days. The protein expression levels of flag-AID and the cell viability were examined through immunoblotting and the Alamar blue assay, respectively. 


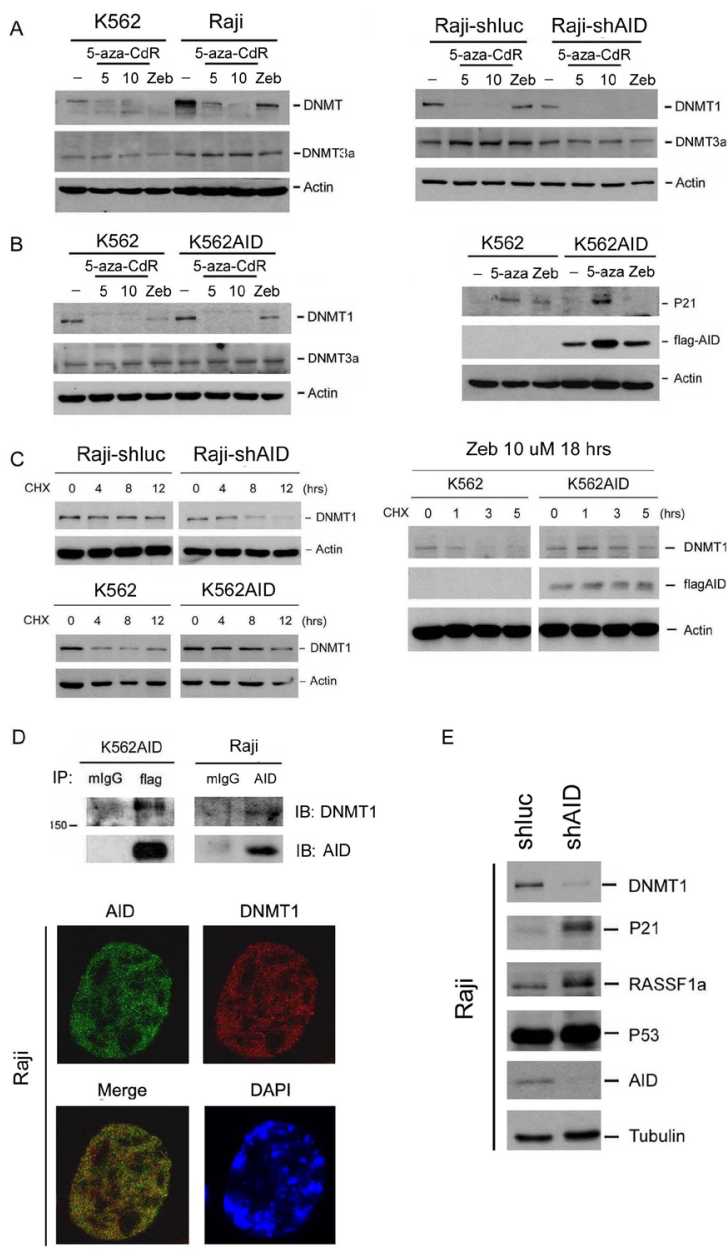

Figure 5: AID blocks DNMT inhibitor-induced degradation of DNMT1 and expression of TSG.s. (A) Left panel: K562 and Raji cells were treated with 5-aza-CdR $(5-10 \mu \mathrm{M})$ or Zeb $(200 \mu \mathrm{M})$ for 24 hrs. Right panel: Raji cells transduced with the shluc or shAID plasmid were treated with 5-aza-CdR (5-10 $\mu \mathrm{M})$ or Zeb (50 $\mu \mathrm{M})$ for $24 \mathrm{hrs}$. (B) Left panel: K562 and pooled K562AID cells were treated with 5-aza-CdR $(5-10 \mu \mathrm{M})$ or Zeb $(10 \mu \mathrm{M})$ for 24 hrs. Right panel: K562 and pooled K562AID cells were treated with 5-aza-CdR $(10 \mu \mathrm{M})$ or Zeb $(10 \mu \mathrm{M})$ for $96 \mathrm{hrs}$. The protein expression levels of DNMTs, p21, flag-AID, and actin were examined through immunoblotting. (C) Left panel: Raji-shluc, Raji-shAID, K562 and K562AID cells were treated with cycloheximide $(20 \mu \mathrm{M})$ for 4, 8, and 12 hrs. Right panel: K562 and K562AID cells were pretreated with Zeb $(10 \mu \mathrm{M})$ for $18 \mathrm{hrs}$ and then exposed to cycloheximide $(20 \mu \mathrm{M})$ for 1,3 , and $5 \mathrm{hrs}$. The protein expression levels of DNMT1, flag-AID, and actin were analyzed through immunoblotting. (D) Upper panel: AID and DNMT1 were coimmunoprecipitated from the total lysate of K562AID4 cells. Flag-AID was precipitated using the anti-flag antibody. Normal mouse IgG (mIgG) was used as the IP control. The protein expression levels of DNMT1 and AID were analyzed through immunoblotting. Lower panel: Raji cells were fixed and stained with anti-AID antibody (green), anti-DNMT1 antibody (red) and DAPI (blue) and subjected to confocal microscopy analysis. (E) Proteins were harvested from Raji-shluc and Raji-shAIDH1 cells, and protein expression levels of DNMT1, p21, Rassf1a, p53, AID, and tubulin were examined through immunoblotting.
$(0.5-5 \mathrm{mg} / \mathrm{kg})$. Zeb inhibited the tumor growth of KCL22engrafted but not KCL22+AID-engrafted mice (suppl. Fig. S5A and Fig. 6C, left panel). On the contrary, 5-aza-CdR effectively inhibited tumor growth of both KCL22 and KCL22+AID grafts (suppl. Fig. S5B and Fig. 6C, right panel), and extended the mouse lifespan (Fig. 6D). These data indicate that 5 -aza-CdR but not Zeb could treat CML$\mathrm{LBC}$ and AID-positive hematopoietic malignancies.

\section{DISCUSSION}

For the past decade, targeted therapy has developed and become dominant for cancer treatment. Nevertheless, cancers are still a cureless disease because of drug resistance, which has never been resolved. Recently, substantial evidences have indicated that AID not only contributes to tumor formation and progression but also causes drug resistance by mutating tumor suppressor genes and oncoproteins $[6,10]$. In addition, AID correlates with poor prognosis $[26,27]$. Therefore, AID might be a potential target for preventing cancer progression and drug resistance. In this study, we found that AID interacted with and may stabilize DNMT1. This association interfered with the ability of a DNMT inhibitor to deplete DNMT1 and induce p21. 5-aza-CdR inhibited nuclear AID expression through ubiquitin-proteasome degradation, thus exerting a cytotoxic effect in AID-positive hematopoietic cancers. In contrast, Zeb effects were limited due to its inability to inhibit AID expression.

Our data have indicated that AID might interact with DNMT1 and stabilize its expression (Fig. 5 and suppl. Fig. S3). However, how AID and DNMT1 interact with each other is currently unclear. DNMT1 transfers methyl groups into the $\mathrm{CpG}$ islands soon after replication to maintain DNA methylation patterns in the newly synthesized single strand. Direct binding of DNMT1 to proliferating cell nuclear antigen (PCNA) in DNA replication sites has been reported[28, 29]. We found the association of AID with PCNA (suppl. Fig. S6). Therefore, PCNA might act as a bridge for AID and DNMT1 interaction. In addition, H3K9 trimethylation by G9a creates a binding platform for HP1, which recruits DNMT1 and increases DNA methylation [30]. It has been reported that AID forms a complex with KRAB domain-associated protein 1 (KAP1) and HP1 during CSR, leading to AID recruitment to switch regions [31]. Therefore, HP1 might also act as a bridge or scaffold for AID and DNMT1 interaction. It has been also shown that post-translational modifications of DNMT1 modulate its stability. SET7/9, a histone methyltransferase, mediates DNMT1 methylation at Lys142 and Lys1094 to induce its proteasomal degradation $[32,33]$. In contrast, DNMT1 can be stabilized by Akt1 phosphorylation at Ser143 which inhibits Lys 142 methylation [33, 34]. PKA phosphorylation of AID at Ser38 is crucial for its activity in CSR [35]. Whether PKA phosphorylation of DNMT1 at Ser143 occurs through AID/DNMT1 complex remains 
to be investigated.

AID-/- ALL show markedly distinct gene expression patterns, with no downregulation of TSGs, such as Rhon, p21, Blnk and TP53 in AID-/- ALL, suggesting that AID may alter gene expression patterns to increase leukemia malignancy [10]. Indeed, the ectopic expression of AID
A

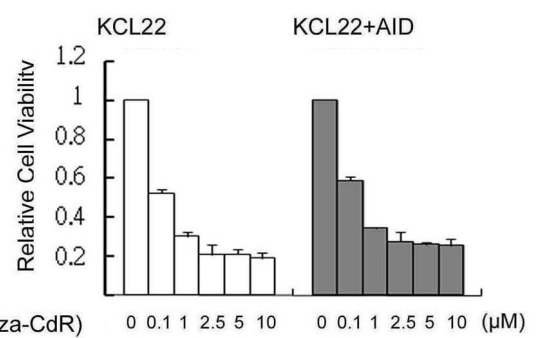

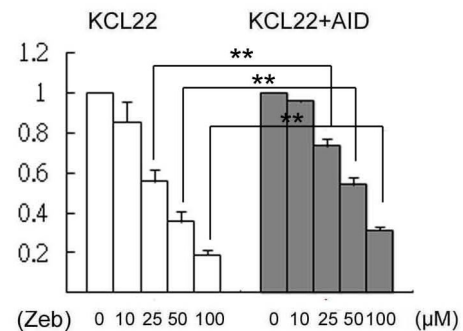

(Zeb) $0102550100 \quad 0102550100 \quad(\mu \mathrm{M})$
B

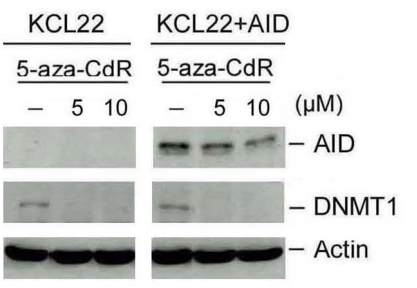

C
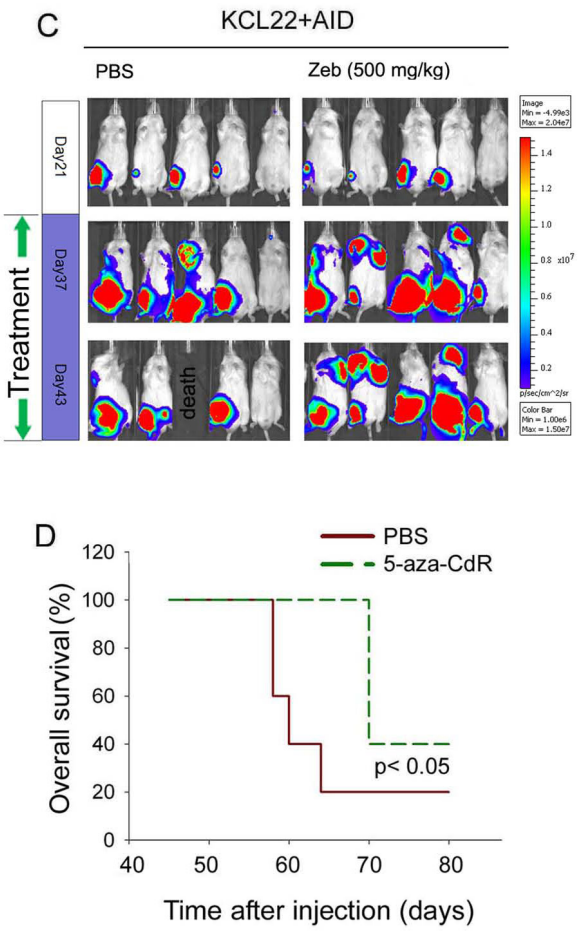
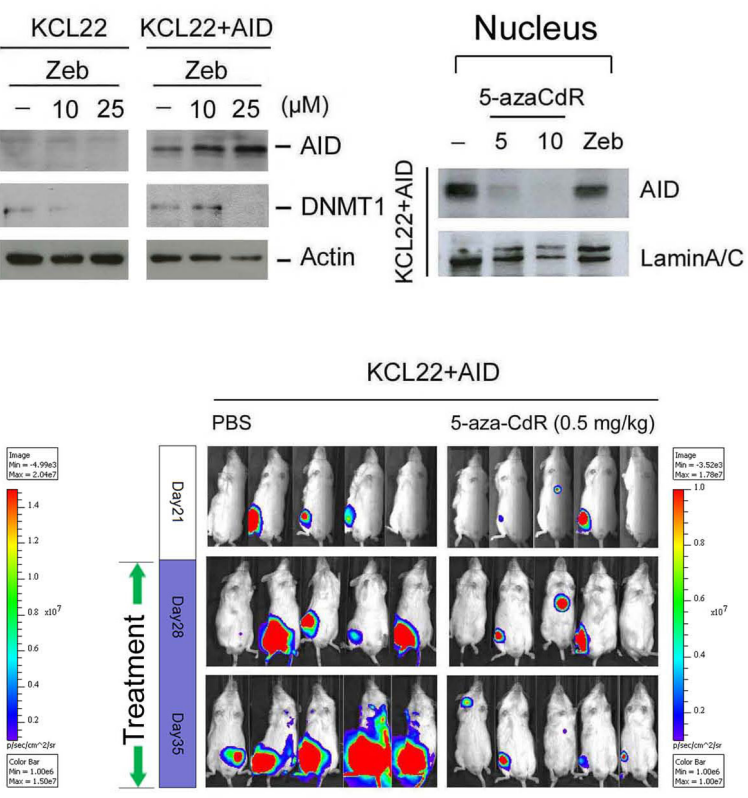

Figure 6: The anti-cancer effect of DNMT inhibitors on tumor-engrafted mice. (A) KCL22 and KCL22+AID cells were treated with 5-aza-CdR $(0.1-10 \mu \mathrm{M}$, left panel) and Zeb (10-100 $\mu \mathrm{M}$, right panel) for 4 days, and cell viability was analyzed with the Alamar blue assay. The error bars represent the mean \pm SD. ${ }^{* *}, \mathrm{p}<0.01$. (B) Left panel: KCL22 and KCL22+AID cells were treated with 5-aza-CdR (5-10 $\mu \mathrm{M}$, left panel) and Zeb (10-25 $\mu \mathrm{M}$, right panel) for $48 \mathrm{hrs}$. The protein expression levels of AID, DNMT1, and actin were analyzed through immunoblotting. Right panel: KCL22+AID cells were treated with 5-aza-CdR $(5$ and $10 \mu \mathrm{M})$ and Zeb $(50 \mu \mathrm{M})$ for 4 days, and the nuclear extracts were harvested. The protein expression levels of AID and lamin A/C were examined through immunoblotting. (C) Left panel: Firefly luciferase-labeled KCL22+AID cells were i.t. injected into NOD/SCID recipient mice. After 21 days, tumor-bearing mice were selected and treated with PBS $(\mathrm{n}=5)$ or Zeb $(500 \mathrm{mg} / \mathrm{kg}, \mathrm{n}=5)$. Cell growth was examined using an in vivo imaging system (IVIS) at day 21,37, and 43. Right panel: Firefly luciferase-labeled KCL22+AID cells were i.t. injected into NOD/SCID mice, and after 21 days, the mice were then treated with PBS $(\mathrm{n}=5)$ or 5 -aza-CdR $(0.5 \mathrm{mg} / \mathrm{kg}, \mathrm{n}=5)$ for 21 days. Cell growth was examined using IVIS at day 21,28 , and 35. (D) Then the overall survival was depicted with Kaplan-Meier analysis. 
in CML cells increased cell survival in vitro and in vivo (suppl. Fig. S4 and S7). Its effect on gene instability could not fully explain this event. Our results showed that silencing AID depleted DNMT1 with the concomitant induction of hypermethylated TSGs, such as p21 and Rassfla, suggesting that differential gene expression in AID+/+ and AID-/- cells may be attributed to DNMT1 levels in cells, in which the methylation status of tumor suppressive genes was altered. In addition, most of B cell lymphoma $\mathrm{t}(14 ; 18)$ translocations occur in the CpG-rich sites where both DNA methylation and AID are required for creating the breakpoints for DNA recombination [36]. The positive association of AID and DNMT1 may be also crucial for $\mathrm{t}(14 ; 18)$ translocations.

AID expression is regulated at the transcriptional and post-transcriptional levels [37]. Our results showed that AID mRNA levels were not significantly affected by 5 -aza-CdR in hematopoietic cancers. Instead, 5-aza$\mathrm{CdR}$ reduced the protein stability of AID by promoting its degradation in the nucleus, suggesting that 5-aza-CdR downregulated AID expression at the post-transcriptional level. Nuclear AID has been shown to be polyubiquitinated and degraded by proteasomes through an unclear mechanism [23]. 5-aza-CdR induces the degradation of DNMT1 through APC/C $\mathrm{C}^{\mathrm{Cdh} 1}$-mediated polyubiquitination [14]. Whether $\mathrm{APC} / \mathrm{C}^{\mathrm{Cdh} 1} \mathrm{E} 3$ ligase is also involved in the AID polyubiquitination and degradation induced by 5-aza-CdR requires investigation. Recently, the E3 ligase RING finger protein 126 (RFN126) has been found for the ubiquitination of AID [38]. Whether 5-aza-CdR could upregulate RFN126 to degrade AID will also be investigated. Alternatively, AID can undergo ubiquitinindependent protein degradation through the REG $\gamma$ proteasome pathway [39]. Although 5-aza-CdR-induced polyubiquitination of AID has been demonstrated, an ubiquitin-independent pathway cannot be excluded.

The structures and metabolisms of 5-azaCdR, 5-aza-CR and Zeb are different. 5-aza-CdR is phosphorylated by deoxycytidine kinase and other kinases into triphosphate, which can be incorporated into newly synthesized DNA [40]. In contrast, 5-aza-CR and Zeb are primarily phosphorylated by uridine-cytidine kinase and other kinases into triphosphates, which are ultimately incorporated into RNA [41]. However, the diphosphate forms of 5-aza-CR and Zeb can also be reduced by ribonucleoside reductase into deoxy-diphosphates, which can be incorporated into DNA [42, 43]. Degradation of DNMT1 by 5-aza-CdR and Zeb demonstrated their incorporation into DNA $[13,44]$. However, only 5-azaCdR could trigger AID degradation. Molecular docking analyses showed that azacytidine but not Zeb-substituted ssDNA could insert into the catalytic site of AID, which might explain the differential effect of 5-aza-CdR and Zeb on AID expression.

5-aza-CR and 5-aza-CdR have been used for the treatment of MDS [45]. They have also been considered powerful candidates for acute myeloid leukemia (AML), CML and ALL [46]. Phase II studies have shown that low doses of 5-aza-CdR exhibit clinical activity against CML, including imatinib-resistant cases $[47,48]$. Furthermore, the combination of 5-aza-CdR and imatinib is well tolerated and active for CML patients in the accelerated

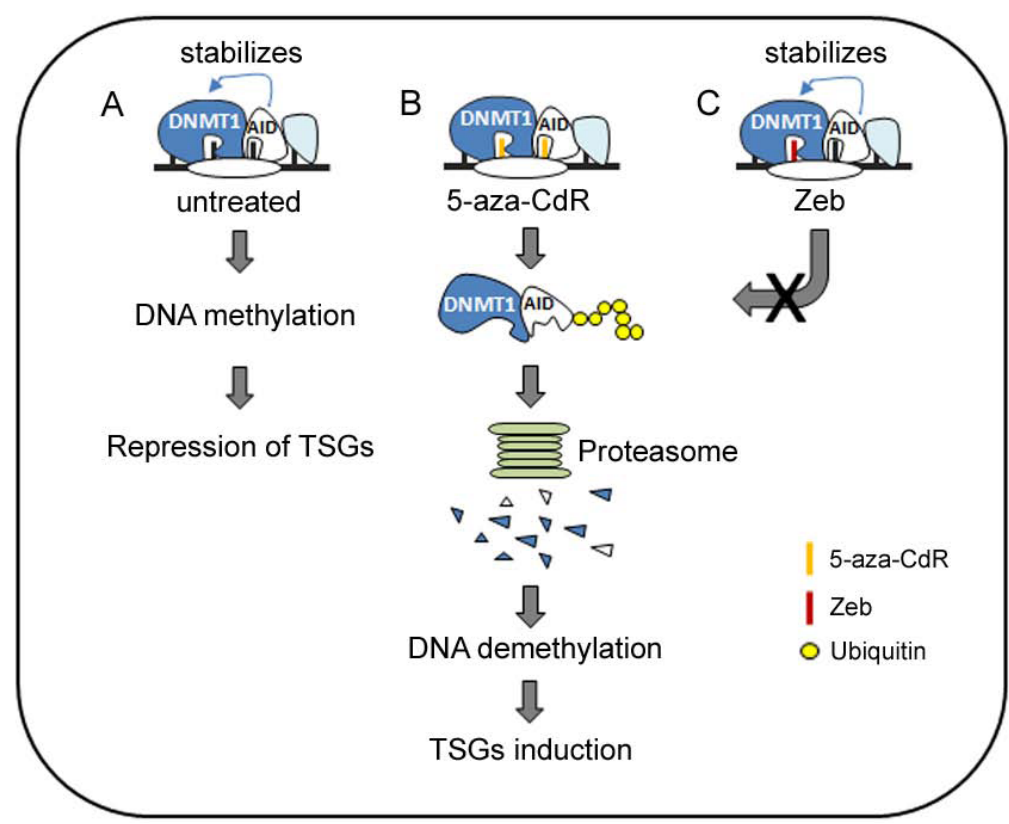

Figure 7: A hypothetical model for the role of AID in the anticancer effect of DNMT inhibitors. (A) AID may co-localize with DNMT1 on DNA and enhance its stability in B-lymphoid malignancies. 5-aza-CdR and Zeb incorporate into DNA. (B) 5-aza-CdR targets the active site of AID and DNMT1 and then destabilizes AID and DNMT1 through the proteasome degradation pathway. Eventually, DNA is demethylated, and TSGs are induced. (C) Because Zeb is unable to bind to the active site of AID, DNMT1 is stabilized and avoids degradation by Zeb. Thus, the anticancer effect of Zeb is inhibited 
or myeloid blastic phase [49]. 5-aza-CdR is currently in a phase I clinical trial for refractory and relapsed ALL (ClinicalTrials.gov identifier: NCT00349596). Because AID contributes to tumorigenesis, imatinib resistance, clonal evolution, and immune evasion in various hematopoietic malignancies $[6,10,50]$, our results provide a novel molecular basis for a new indication of 5-aza-CdR in treating AID-positive hematopoietic cancers.

Taken together, we propose a model for our hypothesis (Fig. 7). The incorporation of DNMT inhibitors into DNA triggers DNMT1 degradation through the proteasomal pathway, resulting in DNA demethylation, TSG induction, and growth arrest. In AID-overexpressing hematopoietic cancer cells, however, AID interacts with and stabilizes DNMT1, which blocks the anticancer effect of Zeb due to its inability to downregulate AID and hinders DNMT1 degradation. In contrast, DNAincorporated 5-aza-CdR can trigger AID degradation through an ubiquitin-proteasome pathway. Thus, 5-azaCdR can effectively degrade DNMT1 to exert its anticancer effect against both AID-positive and AID-negative cells. Our results provide a novel role for the clinical utility of 5-aza-CdR to treat AID-expressing cancers and indicate the crucial concern for the selection of DNMT inhibitors. AID downregulation by DNMT inhibitors, such as 5-aza-CdR, may be beneficial for the treatment of AIDexpressing cancers.

\section{MATERIALS AND METHODS}

\section{Cell culture and construction of stable clones}

AID-overexpressing CML KCL22+AID cells (received from Dr. Markus Müschen, University of California San Francisco) and ALL SUP-B15 cells (obtained from Dr. Hu, Med. Biotech, National Taiwan University) were cultured in IMDM. The Burkett's lymphoma Raji cells (received from Dr. Doong, Department of Microbiology, National Taiwan University) and the CML K562 cells were maintained in RPMI 1640 medium. All media were supplemented with 10\% fetal bovine serum (Gibco), 1\% L-glutamine (Gibco), and 1\% antibiotic:antimycotic solution (Gemini Bio Products), and the cells were incubated at $37^{\circ} \mathrm{C}$ in a humidified incubator containing $5 \% \mathrm{CO}_{2}$. For the generation of stable clones, the pLKO.1-shAID plasmid (purchased from the National RNAiCore Facility, Academia Sinica, Taipei, Taiwan) was transduced into Raji cells through lentivirus infection, and Raji-shAID cells were selected using 100 ng/ml puromycin. The pCMV-3XFLAG-AID plasmid (received from Dr. Riccardo Dalla-Favera, Department of Microbiology, Columbia University) was transfected into K562 cells through electroporation (Nero Transfection System, Invitrogen), and K562AID cells were selected with $1 \mathrm{mg} / \mathrm{ml} \mathrm{G} 418$ (Gibco). 5-azaCR (Sigma Aldrich), 5-aza-CdR (Biovision) and zebularine (Sigma Aldrich) for in vitro experiments were prepared in DMSO at appropriate doses. Zebularine (NSC 309132) for animal studies was provided by the Drug Synthesis and Chemistry Branch, Developmental Therapeutics Program, Division of Cancer Treatment and Diagnosis, National Cancer Institute.

\section{Homology modeling}

The sequence alignment between AID and APOBEC-2 was performed with the ClustalW module using the BLOSUM multiple alignment scoring matrix in Discovery Studio 2.55. The sequence identity and similarity between AID and APOBEC-2 were $31.8 \%$ and $51.0 \%$, respectively. The homology model structure was generated and optimized using MODELLER in Discovery Studio 2.55. The APOBEC-2 structure was obtained from the Protein Data Bank (PDB ID: 2NYT). The structure with the lowest energy score was selected as the final model.

\section{Molecular docking}

Docking simulations of single-stranded DNA were performed using PLANTS 1.2 software. The structure of single-stranded DNA (5'-A-T-azaC-G-3') was built with the "Build and Edit Nucleic Acid" tool in Discovery Studio 2.55. The binding site was set as a sphere with a $40 \AA$ radius centered from the zinc atom to ensure that the entire protein structure was included. All parameters were set to the default settings. Docking simulations of the small molecules Zeb and 5-aza-CdR were performed using the CDOCKER program in Discovery Studio 2.55 with the default parameters. Ligand structures were constructed using the ChemDraw Ultra 12 program and further processed using the Prepare Ligands protocol in Discovery Studio 2.55 to convert to a $3 \mathrm{D}$ structure and assign the ionization state. All parameters were set to the default settings.

\section{Immunofluorescence (IFA) and immunoblotting}

For IFA, the slides with cells were immersed in $2 \%$ paraformaldehyde for $20 \mathrm{~min}$. After fixation, TBST with $1 \%$ goat serum was used to block the slides. The cells were first incubated with the primary antibody antiflag (Sigma Aldrich) or co-incubated with anti-AID (Cell Signaling) and anti-DNMT1 at $4^{\circ} \mathrm{C}$ overnight; then, the secondary antibodies Alexa (488)-labeled anti-mouse and Alexa (647)-labeled anti-goat (Invitrogen) were added and incubated at $25^{\circ} \mathrm{C}$ for $30 \mathrm{~min}$. The signal was observed using a confocal microscope (Leica SP5). For 
immunoblotting, the cells were lysed in sample buffer (50 $\mathrm{mM}$ Tris, $1 \mathrm{mM}$ EGTA, $50 \mathrm{mM} \mathrm{NaF}, 150 \mathrm{mM} \mathrm{NaCl}, 1$ $\mathrm{mM} \mathrm{Na} \mathrm{VO}_{4}, 1 \mathrm{mM} \beta$-glycerophosphate, $8.5 \mathrm{mM}$ sodium pyrophosphate, and $0.5 \%$ Triton X-100). lysates were resolved on a $7.5 \%$ or $13 \%$ sodium dodecyl sulfatepolyacrylamide gel followed by electrotransference to a nitrocellulose membrane (Hybon-C). Then, the membranes were incubated in TBST containing 5\% milk for $1 \mathrm{hr}$. Ultimately, the membranes were incubated with the following primary antibodies: anti-DNMT1, antiDNMT3a, anti-ubiquitin, anti-p21, anti-lamin A/C (all from Santa Cruz Biotechnology), anti-actin (Millipore), anti-tubulin (Sigma-Aldrich) or anti-AID (Cell Signaling).

\section{Immunoprecipitation}

Total cell lysates or nuclear extracts were diluted to $1 \mu \mathrm{g} / \mu \mathrm{l}$, and $200-500 \mu \mathrm{l}$ lysate was incubated with $2-5$ $\mu l$ anti-AID (Cell Signaling) or anti-flag antibody (Sigma Aldrich) overnight at $4{ }^{\circ} \mathrm{C}$. The mixtures containing the lysates and antibody were added to $50 \%$ protein-A beads at a 1:20 ratio (volume) for $1 \mathrm{hr}$ at $4{ }^{\circ} \mathrm{C}$; then, the beads were precipitated at $2000 \mathrm{rpm}$ for $5 \mathrm{~min}$. Then the beads were washed with PBS three times to clear nonspecific proteins. A total of $20 \mu \mathrm{l} \mathrm{PBS}$ and $4 \mu \mathrm{l} 6 \mathrm{X}$ sample dye were mixed with beads to prepare samples for immunoblotting.

\section{In vivo imaging system (IVIS)}

KCL22+AID-luc cells $\left(5 \times 10^{5}\right)$ were intra-tibially (i.t.) injected into NOD/SCID mice. After the xenograft, the mice were imaged at different time points using IVIS (Xenogen, Caliper). The successfully transplanted mice were selected to examine the anticancer effect of the DNMT inhibitors. The treatments were administered weekly on 5 consecutive days as $300 \mu$ i.p. injections of PBS, 5 -aza-CdR $(0.5 \mathrm{mg} / \mathrm{kg})$ or Zeb $(500 \mathrm{mg} / \mathrm{kg})$. D-luciferin (Promega) dissolved in PBS was injected into mice at a dose of $2.5 \mathrm{mg} / \mathrm{mice}$, and the light emission was measured $1 \mathrm{~min}$ later. For anesthesia, 2.5\% isoflurane was administered to the mice via a nose cone. All animal procedures were performed under protocols approved by the Institutional Animal Care and Use Committee of the College of Medicine, National Taiwan University.

\section{RT-PCR}

Total RNA was extracted from cells using $\mathrm{TRI}_{\text {zoL }}$ Reagent (Invitrogen). RNA $(2 \mu \mathrm{g})$ was reverse transcribed into $20 \mu \mathrm{l}$ cDNA by Moloney Murine Leukemia Virus Reverse Transcriptase (M-MLV RT) (Promega) at $42^{\circ} \mathrm{c}$ for $1 \mathrm{hr}$, and then the PCR was performed using Taq polymerase (Geneaid). PCR products were resolved in 1\% agarose gel and visualized by Gel Doc 200 (Bio Rad). The oligonucleotide primers for PCR amplification were as followed: AID, 5'-AGGCAAGAAGACACTCTGGACACC-3' (forward), 5'-GTGACATTCCTGGAAGTTGC-3' (reverse), $\beta$-actin, 5'-TGACGGGGGTCACCCACTGTGCCCATCTA-3' (forward), 5'-CTAGAAGCATTTGCGGGGGACGATGGAGGG-3' (reverse). For quantitative PCR, the cDNA mixed with SYBR Green Master (Roche) was amplified and detected by AVI 7900 (AVI). The oligonucleotide primers for quantitative PCR were as followed: AID, 5'-GGACTTTGGTTATCTTCGCAA-3' (forward), 5'-GTCGGGCACAGTCGTAGC-3' (reverse), $\beta$-actin, 5'-CCAACCGCGAGAAGATGA-3' (forward), 5'-TCCATCACGATGCCAGTG-3' (reverse).

\section{Cell viability assay}

Raji $\left(5 \times 10^{4}\right.$ cells $\left./ \mathrm{ml}\right), \mathrm{K} 562\left(2 \times 10^{4}\right.$ cells $\left./ \mathrm{ml}\right)$ and SUP-B15 $\left(5 \times 10^{5}\right.$ cells $\left./ \mathrm{ml}\right)$ were seeding in 12 well plate, and then treated with DNMT inhibitors at indicated doses for 96 hrs. Cells $(100 \mu \mathrm{l})$ were transferred to 96 well and $10 \mu \mathrm{l}$ alarma blue (Invitrogen) was added to each well. After 2 hrs, cell viability was analyzed by detecting fluorescence. The wavelength at 525-535 nm was used to excite fluorescence. Reference wavelength 535-590 nm was measured on a multiwell plate reader.

\section{Statistical analysis}

Data were analyzed using Student's $t$ test. P values $<0.05$ were considered significant.

\section{ACKNOWLEDGMENTS}

This work was supported by research grants from NSC (to C-C. C.) and the NIH through R01CA139032, R01CA137060, and R01CA157644 (to M.M.).

\section{REFERENCES}

1. Perez-Duran P, de Yebenes VG and Ramiro AR. Oncogenic events triggered by AID, the adverse effect of antibody diversification. Carcinogenesis. 2007; 28(12):2427-2433.

2. Honjo T, Muramatsu M and Fagarasan S. AID: how does it aid antibody diversity? Immunity. 2004; 20(6):659-668.

3. Kinoshita $\mathrm{K}$ and Nonaka $\mathrm{T}$. The dark side of activationinduced cytidine deaminase: relationship with leukemia and beyond. International journal of hematology. 2006; 83(3):201-207.

4. Ramiro AR, Jankovic M, Callen E, Difilippantonio S, Chen HT, McBride KM, Eisenreich TR, Chen J, Dickins RA, Lowe SW, Nussenzweig A and Nussenzweig MC. Role of genomic instability and p53 in AID-induced c-myc-Igh 
translocations. Nature. 2006; 440(7080):105-109.

5. Takizawa M, Tolarova H, Li Z, Dubois W, Lim S, Callen E, Franco S, Mosaico M, Feigenbaum L, Alt FW, Nussenzweig A, Potter M and Casellas R. AID expression levels determine the extent of cMyc oncogenic translocations and the incidence of B cell tumor development. The Journal of experimental medicine. 2008; 205(9):1949-1957.

6. Klemm L, Duy C, Iacobucci I, Kuchen S, von Levetzow G, Feldhahn N, Henke N, Li Z, Hoffmann TK, Kim YM, Hofmann WK, Jumaa H, Groffen J, Heisterkamp N, Martinelli G, Lieber MR, et al. The B cell mutator AID promotes $\mathrm{B}$ lymphoid blast crisis and drug resistance in chronic myeloid leukemia. Cancer Cell. 2009; 16(3):232245.

7. Feldhahn N, Henke N, Melchior K, Duy C, Soh BN, Klein F, von Levetzow G, Giebel B, Li A, Hofmann WK, Jumaa $\mathrm{H}$ and Muschen M. Activation-induced cytidine deaminase acts as a mutator in BCR-ABL1-transformed acute lymphoblastic leukemia cells. The Journal of experimental medicine. 2007; 204(5):1157-1166.

8. Quintas-Cardama A and Cortes J. Molecular biology of bcr-abl1-positive chronic myeloid leukemia. Blood. 2009; 113(8):1619-1630.

9. Garcia-Manero G, Faderl S, O’Brien S, Cortes J, Talpaz $\mathrm{M}$ and Kantarjian HM. Chronic myelogenous leukemia: a review and update of therapeutic strategies. Cancer. 2003; 98(3):437-457.

10. Gruber TA, Chang MS, Sposto R and Muschen M. Activation-induced cytidine deaminase accelerates clonal evolution in BCR-ABL1-driven B-cell lineage acute lymphoblastic leukemia. Cancer Res. 2010; 70(19):74117420 .

11. Komeno Y, Kitaura J, Watanabe-Okochi N, Kato N, Oki T, Nakahara F, Harada Y, Harada H, Shinkura R, Nagaoka $\mathrm{H}$, Hayashi Y, Honjo T and Kitamura T. AID-induced T-lymphoma or B-leukemia/lymphoma in a mouse BMT model. Leukemia. 2010; 24(5):1018-1024.

12. Sorm F, Piskala A, Cihak A and Vesely J. 5-Azacytidine, a new, highly effective cancerostatic. Experientia. 1964; 20(4):202-203.

13. Santi DV, Norment A and Garrett CE. Covalent bond formation between a DNA-cytosine methyltransferase and DNA containing 5-azacytosine. Proc Natl Acad Sci U S A. 1984; 81(22):6993-6997.

14. Ghoshal K, Datta J, Majumder S, Bai S, Kutay H, Motiwala $\mathrm{T}$ and Jacob ST. 5-Aza-deoxycytidine induces selective degradation of DNA methyltransferase 1 by a proteasomal pathway that requires the KEN box, bromo-adjacent homology domain, and nuclear localization signal. Mol Cell Biol. 2005; 25(11):4727-4741.

15. Claus R and Lubbert M. Epigenetic targets in hematopoietic malignancies. Oncogene. 2003; 22(42):6489-6496.

16. Momparler RL, Momparler LF and Samson J. Comparison of the antileukemic activity of 5-AZA-2'-deoxycytidine,
1-beta-D-arabinofuranosylcytosine and 5-azacytidine against L1210 leukemia. Leukemia research. 1984; 8(6):1043-1049.

17. Laliberte J, Marquez VE and Momparler RL. Potent inhibitors for the deamination of cytosine arabinoside and 5-aza-2'-deoxycytidine by human cytidine deaminase. Cancer Chemother Pharmacol. 1992; 30(1):7-11.

18. Chabner BA, Drake JC and Johns DG. Deamination of 5 -azacytidine by a human leukemia cell cytidine deaminase. Biochem Pharmacol. 1973; 22(21):2763-2765.

19. Kim CH, Marquez VE, Mao DT, Haines DR and McCormack JJ. Synthesis of pyrimidin-2-one nucleosides as acid-stable inhibitors of cytidine deaminase. J Med Chem. 1986; 29(8):1374-1380.

20. Carlow D and Wolfenden R. Substrate connectivity effects in the transition state for cytidine deaminase. Biochemistry (Mosc). 1998; 37(34):11873-11878.

21. Prochnow C, Bransteitter R, Klein MG, Goodman MF and Chen XS. The APOBEC-2 crystal structure and functional implications for the deaminase AID. Nature. 2007; 445(7126):447-451.

22. Xu Z, Fulop Z, Wu G, Pone EJ, Zhang J, Mai T, Thomas LM, Al-Qahtani A, White CA, Park SR, Steinacker P, Li Z, Yates J, 3rd, Herron B, Otto M, Zan H, et al. 14-3-3 adaptor proteins recruit AID to 5'-AGCT-3'-rich switch regions for class switch recombination. Nat Struct Mol Biol. 2010; 17(9):1124-1135.

23. Aoufouchi S, Faili A, Zober C, D’Orlando O, Weller S, Weill JC and Reynaud CA. Proteasomal degradation restricts the nuclear lifespan of AID. J Exp Med. 2008; 205(6):1357-1368.

24. Patenaude AM, Orthwein A, Hu Y, Campo VA, Kavli B, Buschiazzo A and Di Noia JM. Active nuclear import and cytoplasmic retention of activation-induced deaminase. Nature structural \& molecular biology. 2009; 16(5):517527.

25. Ito S, Nagaoka H, Shinkura R, Begum N, Muramatsu M, Nakata M and Honjo T. Activation-induced cytidine deaminase shuttles between nucleus and cytoplasm like apolipoprotein B mRNA editing catalytic polypeptide 1 . Proceedings of the National Academy of Sciences of the United States of America. 2004; 101(7):1975-1980.

26. Leuenberger M, Frigerio S, Wild PJ, Noetzli F, Korol D, Zimmermann DR, Gengler C, Probst-Hensch NM, Moch $\mathrm{H}$ and Tinguely $\mathrm{M}$. AID protein expression in chronic lymphocytic leukemia/small lymphocytic lymphoma is associated with poor prognosis and complex genetic alterations. Mod Pathol. 2010; 23(2):177-186.

27. McCarthy H, Wierda WG, Barron LL, Cromwell CC, Wang J, Coombes KR, Rangel R, Elenitoba-Johnson KS, Keating MJ and Abruzzo LV. High expression of activation-induced cytidine deaminase (AID) and splice variants is a distinctive feature of poor-prognosis chronic lymphocytic leukemia. Blood. 2003; 101(12):4903-4908. 
28. Chuang LS, Ian HI, Koh TW, Ng HH, Xu G and Li BF. Human DNA-(cytosine-5) methyltransferase-PCNA complex as a target for p21WAF1. Science. 1997; 277(5334):1996-2000.

29. Leonhardt H, Page AW, Weier HU and Bestor TH. A targeting sequence directs DNA methyltransferase to sites of DNA replication in mammalian nuclei. Cell. 1992; 71(5):865-873.

30. Smallwood A, Esteve PO, Pradhan S and Carey M. Functional cooperation between HP1 and DNMT1 mediates gene silencing. Genes Dev. 2007; 21(10):1169-1178.

31. Jeevan-Raj BP, Robert I, Heyer V, Page A, Wang JH, Cammas F, Alt FW, Losson R and Reina-San-Martin B. Epigenetic tethering of AID to the donor switch region during immunoglobulin class switch recombination. J Exp Med. 2011; 208(8):1649-1660.

32. Esteve PO, Chin HG, Benner J, Feehery GR, Samaranayake M, Horwitz GA, Jacobsen SE and Pradhan S. Regulation of DNMT1 stability through SET7-mediated lysine methylation in mammalian cells. Proceedings of the National Academy of Sciences of the United States of America. 2009; 106(13):5076-5081.

33. Kar S, Deb M, Sengupta D, Shilpi A, Parbin S, Torrisani J, Pradhan S and Patra S. An insight into the various regulatory mechanisms modulating human DNA methyltransferase 1 stability and function. Epigenetics. 2012; 7(9):994-1007.

34. Esteve PO, Chang Y, Samaranayake M, Upadhyay AK, Horton JR, Feehery GR, Cheng X and Pradhan S. A methylation and phosphorylation switch between an adjacent lysine and serine determines human DNMT1 stability. Nature structural \& molecular biology. 2011; 18(1):42-48.

35. Basu U, Franklin A and Alt FW. Post-translational regulation of activation-induced cytidine deaminase. Philos Trans R Soc Lond B Biol Sci. 2009; 364(1517):667-673.

36. Cui X, Lu Z, Kurosawa A, Klemm L, Bagshaw AT, Tsai AG, Gemmell N, Muschen M, Adachi N, Hsieh CL and Lieber MR. Both $\mathrm{CpG}$ methylation and activation-induced deaminase are required for the fragility of the human bcl-2 major breakpoint region: implications for the timing of the breaks in the $t(14 ; 18)$ translocation. Molecular and cellular biology. 2013; 33(5):947-957.

37. Nagaoka H, Tran TH, Kobayashi M, Aida M and Honjo T. Preventing AID, a physiological mutator, from deleterious activation: regulation of the genomic instability that is associated with antibody diversity. Int Immunol. 2010; 22(4):227-235.

38. Delker RK, Zhou Y, Strikoudis A, Stebbins CE and Papavasiliou FN. Solubility-based genetic screen identifies RING finger protein 126 as an E3 ligase for activationinduced cytidine deaminase. Proceedings of the National Academy of Sciences of the United States of America. 2013; 110(3):1029-1034.

39. Uchimura Y, Barton LF, Rada C and Neuberger MS. REG- gamma associates with and modulates the abundance of nuclear activation-induced deaminase. J Exp Med. 2011; 208(12):2385-2391.

40. Momparler RL and Derse D. Kinetics of phosphorylation of 5-aza-2'-deoxyycytidine by deoxycytidine kinase. Biochemical pharmacology. 1979; 28(8):1443-1444.

41. Van Rompay AR, Norda A, Linden K, Johansson M and Karlsson A. Phosphorylation of uridine and cytidine nucleoside analogs by two human uridine-cytidine kinases. Molecular pharmacology. 2001; 59(5):1181-1186.

42. Ben-Kasus T, Ben-Zvi Z, Marquez VE, Kelley JA and Agbaria R. Metabolic activation of zebularine, a novel DNA methylation inhibitor, in human bladder carcinoma cells. Biochem Pharmacol. 2005; 70(1):121-133.

43. Lee T, Karon M and Momparler RL. Kinetic studies on phosphorylation of 5-azacytidine with the purified uridinecytidine kinase from calf thymus. Cancer Res. 1974; 34(10):2482-2488.

44. Schermelleh L, Spada F, Easwaran HP, Zolghadr K, Margot $\mathrm{JB}$, Cardoso $\mathrm{MC}$ and Leonhardt $\mathrm{H}$. Trapped in action: direct visualization of DNA methyltransferase activity in living cells. Nat Methods. 2005; 2(10):751-756.

45. Lubbert M. DNA methylation inhibitors in the treatment of leukemias, myelodysplastic syndromes and hemoglobinopathies: clinical results and possible mechanisms of action. Curr Top Microbiol Immunol. 2000; 249:135-164.

46. Saba HI. Decitabine in myeloid malignancies. Hematology Meeting Reports. 2008; 2(5):1-8.

47. Issa JP, Gharibyan V, Cortes J, Jelinek J, Morris G, Verstovsek S, Talpaz M, Garcia-Manero G and Kantarjian HM. Phase II study of low-dose decitabine in patients with chronic myelogenous leukemia resistant to imatinib mesylate. J Clin Oncol. 2005; 23(17):3948-3956.

48. Kantarjian HM, O'Brien S, Cortes J, Giles FJ, Faderl S, Issa JP, Garcia-Manero G, Rios MB, Shan J, Andreeff M, Keating $M$ and Talpaz M. Results of decitabine (5-aza2'deoxycytidine) therapy in 130 patients with chronic myelogenous leukemia. Cancer. 2003; 98(3):522-528.

49. Oki Y, Kantarjian HM, Gharibyan V, Jones D, O’Brien S, Verstovsek S, Cortes J, Morris GM, Garcia-Manero $\mathrm{G}$ and Issa JP. Phase II study of low-dose decitabine in combination with imatinib mesylate in patients with accelerated or myeloid blastic phase of chronic myelogenous leukemia. Cancer. 2007; 109(5):899-906.

50. Revy P, Muto T, Levy Y, Geissmann F, Plebani A, Sanal O, Catalan N, Forveille M, Dufourcq-Labelouse R, Gennery A, Tezcan I, Ersoy F, Kayserili H, Ugazio AG, Brousse N, Muramatsu M, et al. Activation-induced cytidine deaminase (AID) deficiency causes the autosomal recessive form of the Hyper-IgM syndrome (HIGM2). Cell. 2000; 102(5):565575. 